\title{
Identification of KIF21A Mutations as a Rare Cause of Congenital Fibrosis of the Extraocular Muscles Type 3 (CFEOM3)
}

\author{
Koki Yamada, ${ }^{1,2}$ Wai-Man Chan, ${ }^{2}$ Caroline Andrews, ${ }^{1,2}$ Thomas M. Bosley, ${ }^{3}$ \\ Emin C. Sener, ${ }^{4}$ Johan T. Zwaan, ${ }^{5}$ Paul B. Mullaney, ${ }^{6}$ Banu T. Öztürk, ${ }^{4}$ A. Nurten Akarsu, \\ Louise J. Sabol, ${ }^{8}$ Joseph L. Demer, ${ }^{9}$ Timothy J. Sullivan, ${ }^{10}$ Irene Gottlob, ${ }^{11}$ \\ Peter Roggenkäemper, ${ }^{12}$ David A. Mackey, ${ }^{13}$ Clara E. de Uzcategui, ${ }^{14}$ Nicolas Uzcategui, ${ }^{15}$ \\ Bruria Ben-Zeev, ${ }^{16}$ Elias I. Traboulsi, ${ }^{17}$ Adriano Magli, ${ }^{18}$ Teresa de Berardinis, ${ }^{18}$ \\ Vincenzo Gagliardi, ${ }^{18}$ Sudba Awastbi-Patney, ${ }^{19}$ Marlene C. Vogel, ${ }^{20}$ Joseph F. Rizzo III, ${ }^{21}$ \\ and Elizabeth C. Engle (2,22 $^{1,2}$
}

Purpose. Three congenital fibrosis of the extraocular muscles phenotypes (CFEOM1-3) have been identified. Each repre-

From the Departments of ${ }^{1}$ Genetics and ${ }^{22}$ Neurology, Children's Hospital, Boston, Massachusetts; the ${ }^{2}$ Harvard Medical School, Boston, Massachusetts; the ${ }^{3}$ Division of Neuro-ophthalmology, King Khaled Eye Specialist Hospital, Riyadh, Saudi Arabia; the ${ }^{4}$ Department of Ophthalmology, Ankara Güven Hospital, Ankara, Turkey; the ${ }^{5}$ Department of Ophthalmology, University of Texas Health Science Center, San Antonio, Texas; the ${ }^{6}$ Department of Ophthalmology, Sligo General Hospital, County Sligo, Ireland; the ${ }^{7}$ Gene Mapping Lab, Pediatric Hematology Unit, Department of Pediatrics, Hacettepe University Medical Faculty, Ankara, Turkey; the ${ }^{8}$ Department of Ophthalmology, Geisinger Medical Institute, Danville, Pennsylvania; the ${ }^{9}$ Departments of Ophthalmology and Neurology and the Jules Stein Eye Institute, University of California Los Angeles, Los Angeles, California; the ${ }^{10}$ Royal Children's Hospital, Department of Ophthalmology, University of Queensland, Brisbane, Australia; the ${ }^{11}$ Department of Ophthalmology, University of Leicester, Leicester, United Kingdom; the ${ }^{12}$ University Eye Clinic, Bonn, Germany; the ${ }^{13}$ University of Melbourne, Department of Ophthalmology, Royal Victorian Eye and Ear Hospital, Melbourne, Australia; ${ }^{14}$ Instituto de Otorrinolaringología, San Bernardino, Caracas, Venezuela; the ${ }^{15}$ Children's Hospital of Los Angeles, Doheny Eye Institute, University of Southern California, Los Angeles, California; the ${ }^{16}$ Sheba Medical Center, Sackler School of Medicine, Tel Aviv University, Tel Aviv, Israel; ${ }^{17}$ Cole Eye Institute, the Cleveland Clinic Foundation, Cleveland, Ohio; ${ }^{18}$ Dipartimento di Scienze Oftalmologiche, Facoltà di Medicina e Chirurgia, Università degli Studi di Napoli "Federico II" Napoli, Italy; the ${ }^{19} \mathrm{JKA}$ Institute of Strabismology and Dr. H. L. Patney Memorial Eye Clinic, Rajkot, India; the ${ }^{20}$ Department of Ophthalmology, Hospital de Niños "Roberto del Rio," Santiago, Chile; and the ${ }^{21}$ Department of Ophthalmology, Harvard Medical School and the Massachusetts Eye and Ear Infirmary, Boston, Massachusetts.

Supported by National Eye Institutes Grants R01-EY12498 and R01-EY13583 to (ECE) and P30-HD18655 to Children's Hospital Boston, Mental Retardation Research Center.

Submitted for publication December 31, 2003; revised February 12, 2004; accepted March 3, 2004.

Disclosure: K. Yamada, None; W.-M. Chan, None; C. Andrews None; T.M. Bosley, None; E.C. Sener, None; J.T. Zwaan, None; P.B. Mullaney, None; B.T. Öztürk, None; A.N. Akarsu, None; L.J. Sabol, None; J.L. Demer, None; T.J. Sullivan, None; I. Gottlob, None; P. Roggenkäemper, None; D.A. Mackey, None; C.E. de Uzcategui, None; N. Uzcategui, None; B. Ben-Zeev, None; E.I. Traboulsi, None; A. Magli, None; T. de Berardinis, None; V. Gagliardi, None; S. Awasthi-Patney, None; M.C. Vogel, None; J.F. Rizzo III, None; E.C. Engle, None

The publication costs of this article were defrayed in part by page charge payment. This article must therefore be marked "advertisement" in accordance with 18 U.S.C. $\$ 1734$ solely to indicate this fact.

Corresponding author: Elizabeth C. Engle, Enders 5, Division of Genetics, Children's Hospital, 300 Longwood Avenue, Boston, MA 02115; engle@enders.tch.harvard.edu. sents a specific form of paralytic strabismus characterized by congenital restrictive ophthalmoplegia, often with accompanying ptosis. It has been demonstrated that CFEOM1 results from mutations in KIF21A and CFEOM2 from mutations in $P H O X 2 A$. This study was conducted to determine the incidence of KIF21A and PHOX2A mutations among individuals with the third CFEOM phenotype, CFEOM3.

Methods. All pedigrees and sporadic individuals with CFEOM3 in the authors' database were identified, whether the pedigrees were linked or consistent with linkage to the FEOM1, FEOM2, and/or FEOM3 loci was determined, and the appropriate pedigrees and the sporadic individuals were screened for mutations in KIF21A and PHOX2A.

Results. Twelve CFEOM3 pedigrees and 10 CFEOM3 sporadic individuals were identified in the database. The structures of eight of the pedigrees permitted the generation of meaningful linkage data. KIF21A was screened in 17 probands, and mutations were identified in two CFEOM3 pedigrees. One pedigree harbored a novel mutation $(2841 \mathrm{G} \rightarrow \mathrm{A}, \mathrm{M} 947 \mathrm{I})$ and one harbored the most common and recurrent of the CFEOM1 mutations identified previously $(2860 \mathrm{C} \rightarrow \mathrm{T}, \mathrm{R} 954 \mathrm{~W})$. None of CFEOM3 pedigrees or sporadic individuals harbored mutations in $\mathrm{PHOX} 2 \mathrm{~A}$

Conclusions. The results demonstrate that KIF21A mutations are a rare cause of CFEOM 3 and that $K I F 21 A$ mutations can be nonpenetrant. Although KIF21A is the first gene to be associated with CFEOM3, the results imply that mutations in the unidentified FEOM3 gene are the more common cause of this phenotype. (Invest Ophthalmol Vis Sci. 2004;45:2218-2223) DOI:10.1167/iovs.03-1413

W e have defined three congenital fibrosis of the extraocular muscles phenotypes, CFEOM1-3. In each, affected individuals are born with a nonprogressive ophthalmoplegia affecting extraocular muscles primarily in the oculomotor and/or trochlear nerve distribution. We group these three syndromes with the various forms of Duane syndrome, congenital ptosis, congenital facial palsy, and Moebius syndrome as the congenital cranial dysinnervation disorders (CCDDs), a term we recently proposed for disorders we believe result from aberrant innervation of the ocular and facial musculature. $^{1}$

Individuals with CFEOM1 (OMIM 135700; http://www. ncbi.nlm.nih.gov/Omim/, provided in the public domain by the National Center for Biotechnology Information, Bethesda, MD) are born with bilateral ophthalmoplegia and ptosis, with the primary globe position of each eye infraducted (down- 
ward) and with the inability to raise either above the horizontal midline. The phenotype is quite stereotypic and varies among affected individuals only in the degree of residual normal and aberrant movement within the lower quadrants. Our neuropathologic study demonstrated that CFEOM1 results from absence or hypoplasia of the superior division of the oculomotor nerve and corresponding $\alpha$-motoneurons in the midbrain, with hypoplasia of the levator palpebrae superioris and superior rectus muscles and, presumably, aberrant innervation of other extraocular muscles. ${ }^{2}$ CFEOM1 is the most common of the CFEOM phenotypes and is inherited as a fully penetrant autosomal dominant trait. We have demonstrated that in most pedigrees CFEOM1 maps to the FEOM1 locus on chromosome 12 cen,${ }^{3-6}$ and results from recurrent heterozygous mutations in a developmental kinesin, KIF21A. ${ }^{7}$ Similar to other members of the kinesin superfamily, mouse Kif21a is a motor protein engaged in anterograde axonal transport. ${ }^{8}$ We have identified six different pathogenic KIF21A mutations in 44 (98\%) of 45 CFEOM1 probands. The KIF21A mutations found in CFEOM1 preferentially alter several conserved amino acid residues within the KIF21A stalk region, and we propose they interfere with KIF21A dimerization. We hypothesize that the mutated KIF21A is unable to carry its unidentified cargo from the oculomotor nucleus motoneurons toward the developing neuromuscular junction of the extraocular muscle and that the cargo is critical to the normal development of these axons.

Individuals with CFEOM2 (OMIM 602078) are born with bilateral exotropic ophthalmoplegia and ptosis, with little phenotypic variability. We have identified this recessive disorder in consanguineous pedigrees, mapped it to the FEOM2 locus on $11 \mathrm{q} 13,9$ and shown that it results from homozygous mutations in PHOX2A (ARIX). ${ }^{10,11}$ PHOX2A encodes a homeodomain transcription factor essential to the development of the oculomotor and trochlear motoneurons in mice and zebrafish. ${ }^{12,13}$ Hence, we propose that these cranial nuclei fail to form in CFEOM2 probands.

Individuals with the third CFEOM phenotype, CFEOM3, are those with CFEOM who do not have CFEOM1 or CFEOM2. This includes, for example, individuals who have unilateral CFEOM, have an orthotropic or hypertropic position in primary gaze, or have a primary gaze that is hypotropic but can be elevated above the midline in either eye. CFEOM3 can be inherited as an autosomal dominant trait, and we have identified families in which all affected individuals have CFEOM $3,{ }^{14,15}$ as well as families in which some affected individuals have CFEOM3, and some have CFEOM1. ${ }^{16}$ We define a CFEOM1 pedigree as a CFEOM pedigree in which all affected individuals meet CFEOM1 criteria and a CFEOM3 pedigree as one in which at least ONE affected individual does not meet CFEOM1 criteria (and the pedigree is not CFEOM2). ${ }^{5}$ CFEOM3 pedigrees typically demonstrate broader phenotypic variability than CFEOM1 and CFEOM2 pedigrees, and all contain at least one affected family member with absent or unilateral ptosis, unilateral ophthalmoplegia, noninfraducted primary eye position, and/or the ability to raise at least one globe above the horizontal midline. We have described the linkage analyses of our three large CFEOM3 pedigrees. The phenotype in two maps to a unique locus, FEOM3, on 16qter ${ }^{14,16}$ (OMIM 600638), and the third family's phenotype maps back to the FEOM1 locus ${ }^{17}$ (CFEOM3A; OMIM 607034). The FEOM3 gene has not been identified.

With the identification of KIF21A and PHOX2A as the CFEOM1 and CFEOM2 disease genes, respectively, we are now able to define our CFEOM3 population better. To elucidate the genetic bases of CFEOM3, we identified the CFEOM3 cases in our CCDD database, summarized their clinical phenotypes, determined which CFEOM loci they potentially map to, and screened each pedigree and sporadic case for mutations in both KIF21A and PHOX2A.

\section{Methods}

We identified all CFEOM3 index cases in our participant database using the classification scheme just summarized. ${ }^{5}$ The study was approved by the Children's Hospital Boston institutional review board and informed consent was obtained from participants and/or their guardians. Our methods adhered to the Declaration of Helsinki for research involving human subjects.

Each proband and his/her participating family members were examined and donated a blood sample. High-molecular-weight genomic DNA was extracted from each blood sample according to standard procedures. Linkage analysis using fluorescently labeled microsatellite markers spanning the FEOM1, FEOM2, and FEOM3 loci was performed on those pedigrees with sufficient structure. ${ }^{5}$ KIF21A mutation analysis was conducted by PCR amplification of the 38 KIF21A exons and flanking intron- exon boundaries from genomic DNA of each proband. The amplicons were subjected to analysis by denaturing high-performance liquid chromatography (DHPLC) using a nucleic acid fragment analysis system (WAVE; Transgenomic, Inc., Omaha, NE) and/or to direct DNA sequencing on an ABI 377 DNA sequencer (Applied Biosystems, Foster City, CA) as previously described. ${ }^{7}$ The PCR sequencing and DHPLC primers and conditions are available on request. The three PHOX2A exons and flanking intron-exon boundaries were similarly amplified using our published primer sets ${ }^{10}$ and these amplicons were directly sequenced. Results were compared to normal control individuals. If a mutation was detected in a proband, the participating family members were subsequently screened for the mutation as well.

\section{Results}

\section{Phenotype}

We identified 12 CFEOM3 pedigrees and 10 sporadic CFEOM3 individuals in our CCDD participant database that met our inclusion criteria; their phenotypes are summarized in Table 1. Of the 12 CFEOM3 pedigrees, at least 6 contained one or more affected members with CFEOM1, and at least 3 contained one or more affected members with unilateral ophthalmoplegia. Although there was marked inter- and intrafamilial variability in primary eye position and severity of vertical movement restriction, hypotropic and exotropic globe positions were observed more frequently than the hypertropic and esotropic positions.

Five of the 10 sporadic CFEOM 3 individuals had unilateral disease. Only two of those individuals had ptosis, and in each case the ptosis was ipsilateral to the ophthalmoplegia. Among the five individuals with bilateral ophthalmoplegia, four had bilateral ptosis and one did not. At least two sporadic individuals had an orthotropic primary gaze and one was hypertropic. Most of the sporadic CFEOM3-bearing individuals had absent or severely limited vertical gaze. In contrast, six had normal or only mildly limited horizontal gaze.

\section{Linkage and Haplotype Analysis}

The family structures of 8 of the 12 CFEOM 3 pedigrees permit the generation of potentially meaningful haplotype data at the FEOM1 and FEOM3 loci (Fig. 1 and Table 1). Of these, we have published the clinical description and linkage analysis of the data in pedigrees $\mathrm{BN}$ and $\mathrm{DP}$, which map to the FEOM3 locus, ${ }^{14,16}$ and that in pedigree $\mathrm{BW}$, which maps to FEOM1 with $97 \%$ penetrance and a maximum lod score of $10.8 .{ }^{17}$ Of the 29 affected members of pedigree BW, 18 met CFEOM1 criteria and 11 met those for CFEOM3. The individuals with CFEOM3 had absent ptosis, an orthotropic primary globe position, and/or residual upgaze. Of the five unpublished pedi- 


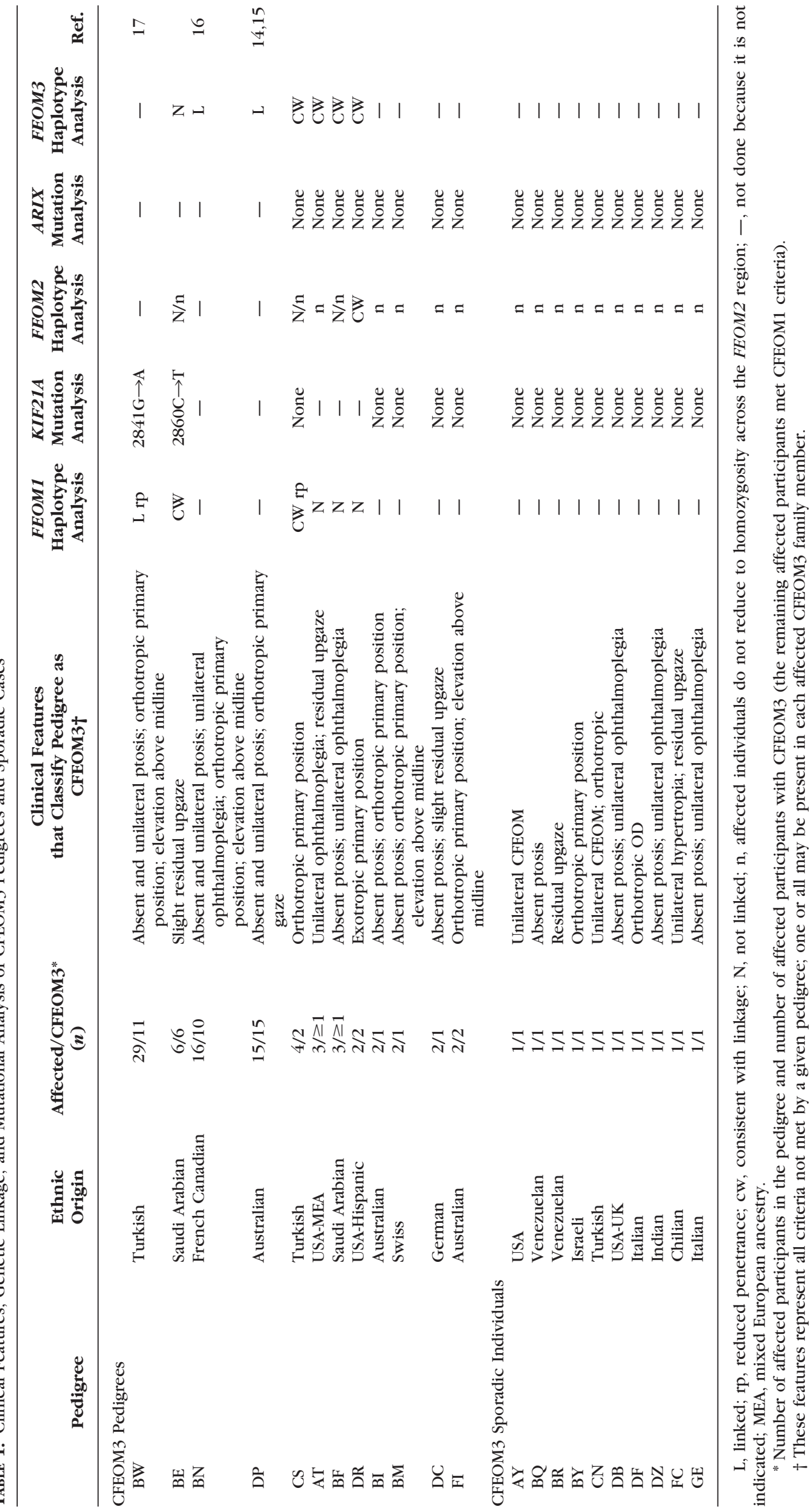


BW

FigURE 1. CFEOM3 pedigrees. Squares and circles symbolize males and females, closed and open squares and circles depict affected and unaffected individuals, respectively, and a diagonal slash through a symbol denotes that the individual is deceased. The cross above and to the right of an individual indicates participation in the study. Pedigrees BN and DP mapped to the FEOM3 locus, have been published, and are not shown. ${ }^{14,16}$ Pedigree $\mathrm{BW}$ has also been published. ${ }^{17}$ Note that pedigree $\mathrm{BW}$ had three consanguineous loops and III:8 and III:19 represent the same individual.

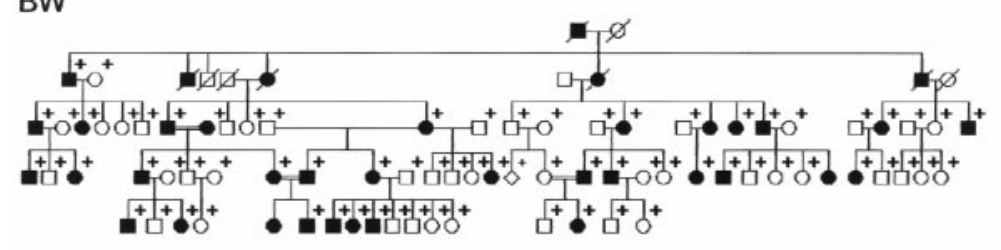

$\mathrm{BE}$
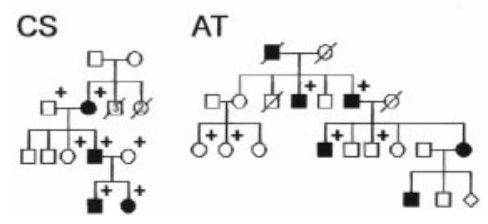

DR

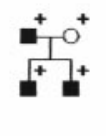

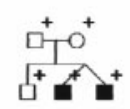

BM

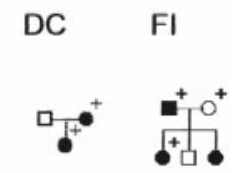

grees (BE, CS, AT, BF, and DR), haplotype analysis of $\mathrm{BE}$ is consistent with linkage to FEOM1 and not to FEOM2 or FEOM3. The six affected members of this family all have bilateral ptosis and infraducted ophthalmoplegia, but are categorized as CFEOM3, because all can raise their eyes above the midline, with $\sim 4 \%$ to $10 \%$ of the normal vertical excursion (Fig. 2). Haplotype analysis of CS is consistent with linkage to FEOM1 with reduced penetrance and FEOM3 with full penetrance. The phenotypes of families AT, BF, and DR are not linked to FEOM1 and are consistent with linkage to FEOM3. The structures of the remaining four pedigrees (BI, BM, DC, and FI) did not permit meaningful haplotype analysis (Fig. 1).

Analysis of the haplotype data for markers spanning the FEOM2 locus reveals that only the phenotype of pedigree DR was consistent with linkage to FEOM2, and this was as a dominant disorder with reduced penetrance. In addition, none of the sporadic individuals or affected members of the pedigrees reduced to homozygosity across the FEOM2 region.

\section{Mutation Analysis}

We screened 17 CFEOM3 probands for mutations in the KIF21A gene, excluding the five pedigrees (BN, DP, AT, BF, and DR) whose phenotypes did not map to FEOM1 and identified mutations in two of the CFEOM3 pedigrees. The affected members of the Turkish pedigree BW, whose phenotype maps to the FEOM1 locus, ${ }^{17}$ harbor a novel KIF21A heterozygous $2841 \mathrm{G} \rightarrow \mathrm{A}$ transition at the third nucleotide position of codon 947 in exon 20, resulting in a methionine-to-isoleucine substitution (M947I; Fig. 3A). The affected members of the consanguineous Saudi Arabian pedigree BE, whose phenotype is consistent with linkage to FEOM1 and none of the other loci, harbor the most common CFEOM1 mutation, ${ }^{7}$ a heterozygous
$2860 \mathrm{C} \rightarrow \mathrm{T}$ transition in exon 21 , leading to an arginine-totryptophan substitution (R954W; Fig. 3B). Each mutation segregates with the CFEOM phenotype in each family and, as predicted by haplotype analysis, ${ }^{17}$ the mutation was also present in the clinically unaffected family member, BW-IV:2. The $2841 \mathrm{G} \rightarrow \mathrm{A}$ change was not found on 210 normal control alleles of diverse ethnicities, including 16 alleles in Turkish pedigrees. The $2860 \mathrm{C} \rightarrow \mathrm{T}$ mutation has been reported in 32 CFEOM1 probands and established as a pathogenic missense mutation. ${ }^{7}$ None of the remaining 15 probands harbors a KIF21A mutation. Of note, a sporadic individual DB carried a rare KIF21A single-nucleotide polymorphism, 3641C $\rightarrow$ G (P1214R), that we identified earlier in two members of a large CFEOM1 pedigree with the common $2860 \mathrm{C} \rightarrow$ T mutation. $^{7}$

To determine whether any cases of CFEOM3 result from mutations in PHOX2A, we sequenced the three coding exons in all probands, except for $\mathrm{BW}$ and $\mathrm{BE}$ who harbored KIF21A mutations and $\mathrm{BN}$ and $\mathrm{DP}$ whose phenotypes mapped to FEOM3. No PHOX2A mutations were detected.

\section{Discussion}

We identified our cohort of CFEOM3 pedigrees and sporadic individuals from our CCDD database and screened them for linkage to the FEOM loci and for mutations in KIF21A and $P H O X 2 A$. We identified pathogenic KIF21A missense mutations in $2(9 \%)$ of the 22 CFEOM3 probands in our database, establishing that mutations in KIF21A are a rare cause of CFEOM 3 and confirming the genetic heterogeneity of CFEOM3

Pedigree BW is a large Turkish family whose CFEOM phenotype was strikingly different from the CFEOM1 pedigrees we
FIGURE 2. Photographs of an affected member of pedigree $\mathrm{BE}$ after ptosis surgery with eyes in primary position (center) and eight fields of gaze. Note the participant's ability to raise both eyes slightly above the midline. Her chin-up face position improved significantly after correction of the ptosis. Similarly, other affected family members' chin-up position also seemed to correlate best with their degree of ptosis, suggesting that their primary globe positions were nearly neutral. Horizontal and down gaze were relatively full.
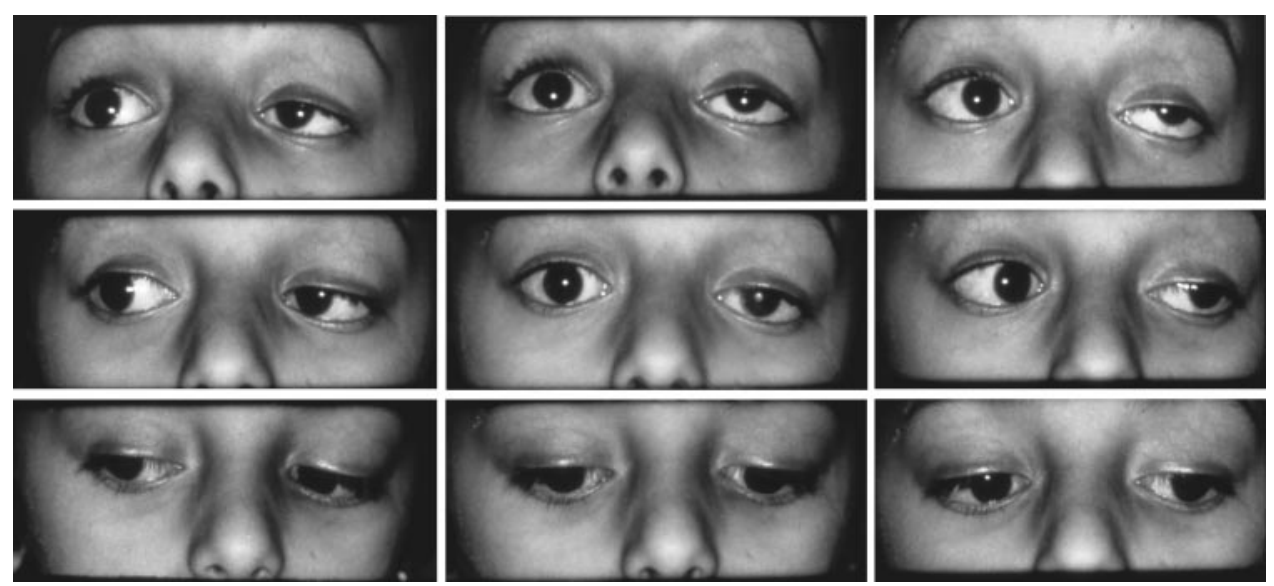
A

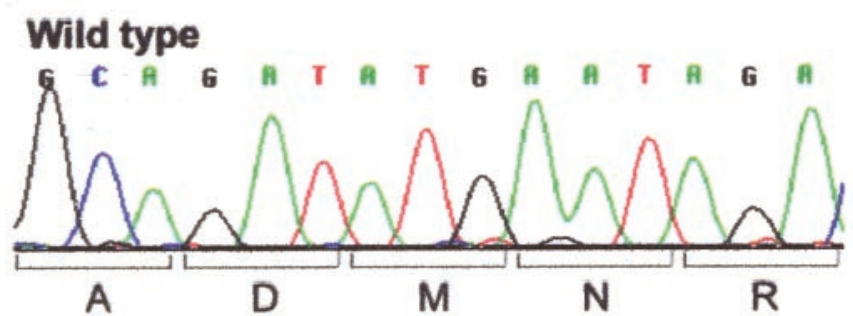

A Novel Mutation

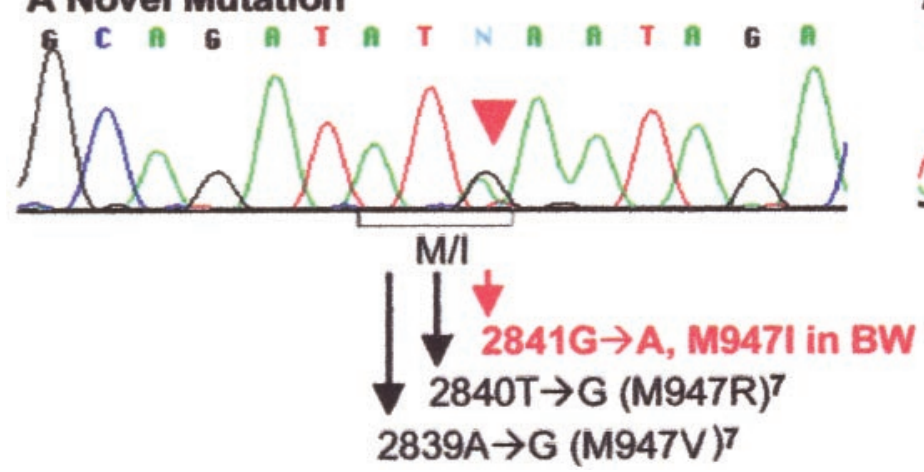

B

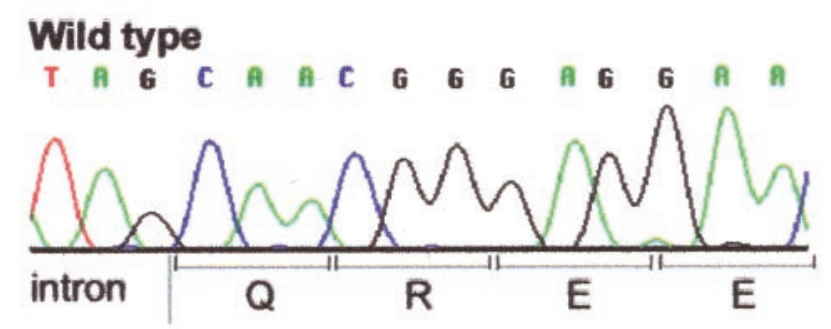

A Recurrent Mutation

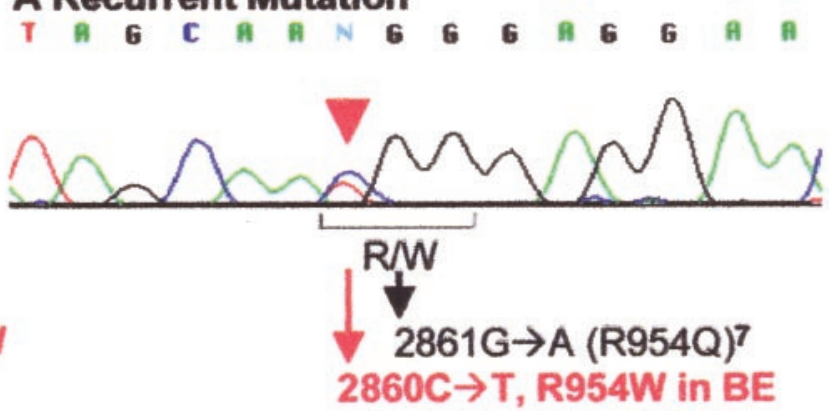

FIGURE 3. Sequence chromatographs of unaffected control individuals (top) and individuals with CFEOM3 (bottom) showing heterozygous mutations in KIF21A. (A) Members of pedigree BW harbored a novel transition, 2841G $\rightarrow$ A (M947I) (red triangle). Combined with the previously reported mutations, indicated by black arrows beneath the chromatographs, we have now identified three different point mutations that alter nucleotide positions 1, 2, and 3 of codon 947. (B) Members of pedigree BE harbor the common CFEOM1 mutation, 2860C $\rightarrow$ T (R954W) (red triangle). Earlier, we identified the second most common CFEOM1 mutation, which alters nucleotide position 2 of codon 954 , indicated by the black arrow below the chromatograph.

have described, yet the phenotype maps to the FEOM1 locus. ${ }^{17}$ Of interest, we found that the affected members of this pedigree harbored a novel $2841 \mathrm{G} \rightarrow \mathrm{A}$ mutation in KIF21A that alters the third nucleotide position of codon 947 (M947I). We have previously identified CFEOM1 mutations at nucleotide positions 1 and 2 of this codon. ${ }^{7}$ We found a heterozygous $2839 \mathrm{~A} \rightarrow \mathrm{G}$ transition (M947V) at the first nucleotide position in a small CFEOM1 pedigree with only three affected individuals and a heterozygous de novo $2840 \mathrm{~T} \rightarrow \mathrm{G}$ transversion (M947R) at the second nucleotide position in an individual with sporadic CFEOM1. Both the M947I and the M947V mutations maintain nonpolar aliphatic R groups at codon 947 and are the only disease-causing conservative KIF21A amino acid substitutions we have identified. The M947I substitution is also the first KIF21A mutation we have detected that results in CFEOM with variable severity and incomplete penetrance. The occurrence of a CFEOM3 rather than CFEOM1 phenotype in pedigree $\mathrm{BW}$ may result in part from environmental factors and genetic background. Of note, however, we have only identified four CFEOM1 individuals with mutations at codon 947 (three familial and one sporadic), and we know that a subset of individuals within the CFEOM3 pedigree $\mathrm{BW}$ express the CFEOM1 phenotype. ${ }^{17}$ Therefore, it is possible that mutations in codon 947 or mutations that result in conservative amino acid changes can cause a more variable and milder CFEOM phenotype than KIF21A mutations at the other nucleotide positions. Mutation studies of additional CFEOM pedigrees will help to determine whether such phenotype-genotype predictions are possible.

We identified a KIF21A mutation in only one additional CFEOM3 pedigree, the Saudi Arabian pedigree BE. Of note, this pedigree harbored the most common CFEOM1 "hotspot" mutation, $2860 \mathrm{C} \rightarrow \mathrm{T}$, found in $32 \mathrm{CFEOM} 1$ families and sporadic cases and accounting for $72 \%$ of all KIF $21 A$ mutation events we have identified to date. ${ }^{7}$ In retrospect, we recognize that all six affected members of pedigree BE shared a similar phenotype that resembles CFEOM1 more closely than it resembles the phenotypes found in the affected members of pedigree BW. All six affected family members had bilateral infraducted ophthalmoplegia and ptosis. Their degree of infraduction was small compared with most CFEOM1 pedigrees, and they are classified as CFEOM3 because they could elevate their eyes slightly above the midline. The ability to elevate their eyes could result from a small degree of residual function of the superior rectus, or possibly from the function of an aberrantly inserted superior oblique or horizontal rectus muscle(s). Additional genetic studies to determine the frequency with which this common KIF21A mutation occurs in CFEOM3 individuals, combined with clinical studies documenting the degree of vertical excursion in CFEOM1 individuals harboring KIF21A mutations, should help determine whether it would be appropriate to broaden our clinical definition of CFEOM1 to permit a small degree of globe elevation above the horizontal. If this were done, then pedigree $\mathrm{BE}$ and sporadic individual BR (in whom we did not identify a KIF21A mutation) would be reclassified as CFEOM1.

Combining these new findings with our published data, we have now identified CFEOM1- and CFEOM3-causing mutations in seven nucleotide positions at four distinct amino acid residues within three exons of KIF21A. The small number of altered residues enhances the feasibility of cost-effective KIF21A mutation detection in patients with CFEOM, despite the large number of KIF21A exons. Currently, we screen KIF21A exons 8,20 , and 21 before a more exhaustive search of the remaining 35 exons.

None of the CFEOM3 pedigrees or sporadic cases had mutations in the CFEOM2 gene, $P H O X 2 A$. This is similar to our finding that none of our CFEOM1 cases harbored $\mathrm{PHOX} 2 \mathrm{~A}$ 
mutations, ${ }^{5}$ and reinforces CFEOM2 as the sole CFEOM phenotype caused by mutations in this gene.

We did not identify KIF21A or PHOX2A mutations in the remaining $10 \mathrm{CFEOM} 3$ pedigrees or in any of our sporadic CFEOM3 individuals. Two of these CFEOM3 pedigrees were linked to FEOM3, four were consistent with linkage to FEOM3, and four were indeterminate. Therefore, we anticipate that many of these pedigrees and a subset of the sporadic cases harbor mutations in the unidentified FEOM3 gene.

Additional genetic studies, including the identification of FEOM3 and other associated gene(s), will facilitate the molecular genetic-based diagnosis of CFEOM phenotypes and will help us to define better the CFEOM1 and CFEOM3 phenotypes and to determine the feasibility of phenotype-genotype correlations. In addition, future molecular investigations should lead to a better understanding of the selective vulnerability of the oculomotor and/or trochlear motoneuron unit by the mutations in KIF21A, PHOX2A, and the unidentified FEOM3 gene(s).

\section{Acknowledgments}

The authors thank all the patients and their families for participating in the study, Rae R. Fellows and Don L. Bremer for referring patients, and Maria P. Rogines-Velo-Sardi and Carlos Miranda for helpful discussions.

\section{References}

1. Gutowski NJ, Bosley TM, Engle EC. 110th ENMC International Workshop: The congenital cranial dysinnervation disorders (CCDDs). Naarden, The Netherlands, 25-27 October, 2002. Neuromuscul Disord. 2003;13:573-578.

2. Engle EC, Goumernov B, McKeown CA, et al. Oculomotor nerve and muscle abnormalities in congenital fibrosis of the extraocular muscles. Ann Neurol. 1997;41:314-325.

3. Engle EC, Kunkel LM, Specht LA, Beggs AH. Mapping a gene for congenital fibrosis of the extraocular muscles to the centromeric region of chromosome 12. Nat Genet. 1994;7:69-73.

4. Engle EC, Marondel I, Houtman WA, et al. Congenital fibrosis of the extraocular muscles (autosomal dominant congenital external ophthalmoplegia): genetic homogeneity, linkage refinement, and physical mapping on chromosome 12. Am J Hum Genet. 1995; 57:1086-1094.

5. Engle EC, McIntosh N, Yamada K, et al. CFEOM1, the classic familial form of congenital fibrosis of the extraocular muscles, is genetically heterogeneous but does not result from mutations in ARIX. BMC Genet. 2002;3:3.

6. Uyama E, Yamada K, Kawano H, et al. A Japanese family with FEOM1-linked congenital fibrosis of the extraocular muscles type 1 (CFEOM1) associated with spinal canal stenosis and refinement of the FEOM1 critical region. Neuromuscul Disord. 2003;13:472478.

7. Yamada K, Andrews C, Chan WM, et al. Heterozygous mutations of the kinesin KIF21A in congenital fibrosis of the extraocular muscles type 1 (CFEOM1). Nat Genet. 2003;35:318-321.

8. Marszalek JR, Weiner JA, Farlow SJ, Chun J, Goldstein LS. Novel dendritic kinesin sorting identified by different process targeting of two related kinesins: KIF21A and KIF21B. J Cell Biol. 1999;145: $469-479$.

9. Wang SM, Zwaan J, Mullaney PB, et al. Congenital fibrosis of the extraocular muscles type 2, an inherited exotropic strabismus fixus, maps to distal 11q13. Am J Hum Genet. 1998;63:517-525.

10. Nakano M, Yamada K, Fain J, et al. Homozygous mutations in ARIX(PHOX2A) result in congenital fibrosis of the extraocular muscles type 2. Nat Genet. 2001;29:315-320.

11. Yazdani A, Chung DC, Abbaszadegan MR, et al. A novel PHOX2A/ ARIX mutation in an Iranian family with congenital fibrosis of extraocular muscles type 2 (CFEOM2). Am J Ophthalmol. 2003; 136:861-865.

12. Pattyn A, Morin X, Cremer H, Goridis C, Brunet JF. Expression and interactions of the two closely related homeobox genes Phox 2a and Phox2b during neurogenesis. Development. 1997;124:40654075 .

13. Guo S, Brush J, Teraoka H, et al. Development of noradrenergic neurons in the zebrafish hindbrain requires BMP, FGF8, and the homeodomain protein soulless/Phox2a. Neuron. 1999;24:555566.

14. Mackey DA, Chan WM, Chan C, et al. Congenital fibrosis of the vertically acting extraocular muscles maps to the FEOM3 locus. Hum Genet. 2002;110:510-512.

15. Gillies W, Harris A, Brooks A, Rivers M, Wolfe R. Congenital fibrosis of the vertically acting extraocular muscles: a new group of dominantly inherited ocular fibrosis with radiologic findings. $O p b$ thalmology. 1995;102:607-612.

16. Doherty E, Macy M, Wang S, Dykeman C, Melanson M, Engle E. CFEOM3: a new extraocular congenital fibrosis syndrome that maps to 16q24.2-q24.3. Invest Opbthalmol Vis Sci. 1999;40:16871694.

17. Sener EC, Lee BA, Turgut B, Akarsu AN, Engle EC. A clinically variant fibrosis syndrome in a Turkish family maps to the CFEOM1 locus on chromosome 12. Arch Ophthalmol. 2000;118:10901097. 